\title{
Report of a Mississippi hospital's experience with rapid conversion to robotic-assisted gynecological
}

\section{surgery}

\begin{abstract}
The use of robotic-assisted gynecological surgery has grown at an unprecedented rate since its approval by the United States Food and Drug Administration in 2005. This rapid adoption of new technology has been demonstrated at Merit Health Woman's Hospital, a medical and surgical facility in Flowood, Mississippi, outside of Jackson. Woman's Hospital operates 60 patient beds with five operating rooms and began its robotic-assisted gyn surgical program in December 2010. The facility now has three fully functional daVinci Si robotic systems utilized by 27 gynecologists as well as by general surgeons and urologists. Through the end of 2014, a total of 1943 robotic-assisted total laparoscopic hysterectomies (with or without removal of the adnexa) were performed, nearly eliminating laparotomy procedures. Robotic-assisted surgery also replaced total laparoscopic surgical techniques performed "straight-stick" or rigid (i.e., without the utilization of wristed laparoscopic instruments) and has nearly replaced vaginal hysterectomies. Average length of procedure defined as "skin-to-skin" or start of first incision to complete skin closure was less for robot-assisted total laparoscopic hysterectomy (RATLH) than total abdominal hysterectomy (TAH), each with or without bilateral salpingo-oophorectomy (BSO).

Physicians have found robotic-assisted techniques to be less physically taxing. Likewise, among Woman's Hospital patients surveyed, patient satisfaction remained high as minimally invasive robotic techniques replaced laparotomy. The surgical site patient infection rate has fallen from $3.9 \%$ of total hysterectomy cases performed in 2009 prior to initiation of our robotic program to an even lower $1.3 \%$ in 2014 after nearly all hysterectomies were performed using robot-assisted techniques. Quality control standards regarding operating times and surgical credentialing are enforced in the program.
\end{abstract}

Keywords: robotic-assisted gynecological surgery, davinci, merit health woman's hospital, laparotomy, infection rates, surgeon credentialing
Volume 2 Issue I - 2015

\author{
Darden North \\ Jackson Healthcare for Women, USA
}

Correspondence: Darden North, Jackson Healthcare for Women, 29I East Layfair Drive, Flowood, MS 39232, PA, USA, Tel:601.936.9190,601.955.1197, Email DardenN@aol.com

Received: January 0I, 2015 | Published: February 14, 2015
Abbreviations: RATLH, robot-assisted total laparoscopic hysterectomy; TAH, total abdominal hysterectomy; BSO, bilateral salpingo-oophorectomy; LAVH, laparoscopic-assisted vaginal hysterectomies; TLH, total laparoscopic hysterectomy

\section{Introduction}

The use of robotic-assisted gynecological surgery has grown at an unprecedented rate since its approval by the United States Food and Drug Administration in April 2005. Reports of the Nationwide Inpatient Sample of the Agency for Healthcare Research and Quality indicate that the number of robot-assisted hysterectomies has increased dramatically. ${ }^{1}$ Merit Health Woman's Hospital, a medical and surgical facility in Flowood, Mississippi, located just outside of Jackson, is licensed for 109 beds but operates 60 and has lead in this technological revolution.

The rapid adoption of robotic-assisted laparoscopic surgery among gynecologists has been attributed to several factors. Depending on the level of training and experience of individual physicians, one factor may be the ease of acquiring robotic-assisted laparoscopic surgical skills over traditional or "straight-stick," non-wristed, laparoscopic techniques. The similarity of robotic surgical views of the abdomen and pelvis (both multi-site and single-site platforms) to the traditional open surgical view optimizes this modern surgical approach. (For the purpose of this article, an "open" surgical case or approach is defined as one completed through a full-thickness abdominal laparotomy skin incision, either transverse or vertical and of a length greater than one to two centimeters.) Also the higher laparoscopic camera magnification (20X) and 3-D high-definition resolution available with, for example, the daVinci robotic camera system generally affords better visibility than simple laparoscopy alone. This enhancement is complemented by a full array of wristed instrumentation (in multi-site daVinci platforms) that may allow for the completion of more technically demanding cases than would have been possible in many non-robotic, "straight-stick" laparoscopic venues. Without the benefits of the improved camera and instrumentation, conversion to laparotomy from laparoscopy is many times avoided in robotic-assisted surgery. In most instances, robotic-assisted laparoscopy is less physically demanding on the surgeon by avoiding muscle fatigue of the upper and lower extremities as the robotic arms stabilize the laparoscopic surgical instruments and camera. A surgeon is able to perform fine laparoscopic dissection, tissue cauterization, and tissue division while sitting at a console control.

The increased utilization of robotic surgery has paralleled extensive commercial marketing of the technology not only to physicians and surgical facilities but also to medical consumers and potential patients. Patients have been quick to appreciate the enhanced cosmesis realized through greater use of laparoscopy over laparotomy as well as the 
decreased need for narcotic pain relief post-op. This decreased recovery morbidity has the potential for reducing recuperation periods and thus more quickly returning patients to work, hobbies, or family. Surgical facilities have generally reported decreased post-operative infection rates with robotic-assisted gyn surgery versus laparotomy, predominately due to the smaller laparoscopic skin incisions. However, improved return to ambulation with the use of minimally invasive laparoscopy potentially lowers risks of atelectas is and venous thromboembolic disease, both etiologies of febrile morbidity. ${ }^{2}$

\section{Case presentation}

The surgical case history at Merit Health Woman's Hospital reflects this dramatic increase in the utilization of robotic technology. Our initial daVinci Si surgical robotic system from Intuitive Surgical was installed in late December 2010. Primary surgeon credentialing for robotic cases includes prerequisite observation of such cases before completing an intensive hands-on training seminar, followed by successfully performing a minimum number of proctored cases. To maintain surgical privileges at Merit Health Woman's Hospital, all credentialed robotic-assisted surgeons must perform at least 20 cases yearly at Woman's or surrounding facilities.

Since the first of now three daVinci Si robots was installed within our five operating rooms, the types of procedures performed have predominately included robotic-assisted total laparoscopic hysterectomies (RATLH) with or without removal of the adnexa, myomectomies (prior to the recall and lack of availability of power morcellators), salpingo-oophorectomy or oophorectomy alone, and ovarian cystectomies. Other specialties, such as general surgery and urology, have performed smaller numbers of cases at Woman's including robot-assisted cholecystectomies, sacrocolpoplexies, and even two prostatectomies.

Between 2006 and 2014 the number of hysterectomies performed at Merit Health Woman's Hospital was reviewed. While the interest here in minimally invasive surgical techniques has mirrored national trends, the conversion from open cases has been quite rapid. Table 1 enumerates the methods of hysterectomy provided by Merit Health Woman's Hospital from 2006 - 2014.

Table I Methods of Hysterectomy provided at Merit Health Women's Hospital 2006-20I4

\begin{tabular}{|c|c|c|c|c|c|c|c|c|c|}
\hline Procedure & 2006 & 2007 & 2008 & 2009 & 2010 & 2011 & 2012 & 2013 & 2014 \\
\hline TAH & $262(63.0)$ & $208(45.0)$ & $163(38.3)$ & I5I (3I.5) & $109(23.0)$ & $48(7.7)$ & $26(4.5)$ & $15(3.3)$ & $26(5.2)$ \\
\hline Vag Hyst & $57(13.7)$ & $64(13.9)$ & $53(12.4)$ & $39(8.1)$ & $32(6.8)$ & $18(2.9)$ & II (I.9) & $13(2.9)$ & $2(0.4)$ \\
\hline LAVH & $97(23.3)$ & 170 (36.8) & $157(36.9)$ & $160(33.3)$ & $139(29.4)$ & $30(4.8)$ & $23(3.9)$ & $7(1.5)$ & 0 \\
\hline TLH & 0 & $20(4.3)$ & $53(12.4)$ & $130(27.1)$ & $190(40.2)$ & 0 & 0 & I (0.2) & 0 \\
\hline RATLH & 0 & 0 & 0 & 0 & $3(0.6)$ & $525(84.5)$ & $524(89.7)$ & $416(92.0)$ & 475 (94.4) \\
\hline Total Procedures & 416 & 462 & 426 & 480 & 473 & 621 & 584 & 452 & 503 \\
\hline $\begin{array}{l}\text { Annual Infection } \\
\text { Rate }\end{array}$ & 1.33 & 2.65 & 7.97 & 3.16 & 4.9 & 4.26 & 0 & 1.8 & 1.3 \\
\hline
\end{tabular}

The annual number of each type of hysterectomy is listed, followed in parenthesis by its percentage of the yearly total

Of the 416 hysterectomy cases performed in 2006, $262(63.0 \%)$ were total abdominal hysterectomies (TAH) with or without removal of the adnexa, $57(13.7 \%)$ vaginal hysterectomies (VH), and 97 (23.3\%) laparoscopic-assisted vaginal hysterectomies (LAVH). Upon the introduction in 2007 of Total Laparoscopic Hysterectomy (TLH) with or without removal of the adnexa-using only non-robotic, rigid laparoscopic instruments-only $20 \mathrm{TLH}$ cases $(4.3 \%)$ were performed by the end of the year out of a total of 462 hysterectomies. By the close of 2010when robotic surgery was introduced at Woman's Hospital, the annual number of TAHs fell to $109(23.0 \%)$ of the total 473 hysterectomy cases. In comparison, the number of TLH cases grew in 2010 to $190(40.2 \%)$. By the end of 2011 nearly all active staff gynecological surgeons were certified as robotic surgeons. During the first full 12 months of availability of the daVinci robotic surgical system in 2011, there were no TLHs recorded, only 48 (7.7\%) of 621 total hysterectomy cases were performed via the TAH route, and $525(84.5 \%)$ were completed robotically. Despite a decrease in total hysterectomies in 2012 as compared to 2011, the percentage of robotic hysterectomies with or without the removal of the adnexa (RATLH) increased to $89.7 \%$ in 2012 versus $84.5 \%$ in 2011 . A total of 452 hysterectomies were performed in 2013:92.0\% were RATLHs, $0.2 \%$ were TLHs, $3.3 \%$ were TAHs, $2.9 \%$ were $\mathrm{VHs}$, and $1.5 \%$ were LAVHs. Of the total number of hysterectomies performed in 2014, $94.4 \%$ were robotic ( 475 of 503 ) while $5.2 \%$ were open ( 26 of 503 ) and $0.4 \%$ were vaginal ( 2 of 503 ). There were no LAVHs or TLHs performed in 2014.

Critical discussions regarding the growing use of robotic-assisted laparoscopic minimally invasive surgery have included comparisons of operating times ("skin-to-skin") between TAH, TLH, and RATLH cases as well as differences in post-op infection rates. Length of operations and surgical suite turnover times between cases were closely monitored in 2014 at Merit Health Woman's Hospital and manually recorded by the registered nurse in charge of the operating room during each procedure. Length of surgical case completion for our RATLH procedures averaged one hour and eleven minutes ( 1.18 hours) in comparison to two hours and ten minutes ( 2.17 hours) for TAH procedures. Of the RATLHs performed in 2014, twelve surgeons were primary on 15 or more cases for a total of $82.1 \%$ of all procedures while of those 12 gynecologists, six performed 30 or more for a total of $55.5 \%$ of all cases.

Regarding surgical site infections, the post-operative infection rate fell from an already low $3.16 \%$ in 2009 to $0.00 \%$ in $2012,1.8 \%$ in 2013 , and most recently $1.3 \%$ at the end of 2014 as robotic-assisted procedures replaced open techniques. The readmission rate for postop infections following RATLH in 2013 was $1.4 \%$. Five of the six readmissions of that total were for management of deep surgical site infections and one was related to an infected skin incision. ${ }^{3}$ 
To determine patient satisfaction with Woman's Hospital, discharged patients have been surveyed by Press Ganey and now Health Stream. The data base information continues to find an $80 \%$ or greater patient satisfaction rate as minimally invasive robotic techniques replace laparotomy. ${ }^{4}$

\section{Discussion}

Our experience with robotic-assisted minimally-invasive gynecological surgery at Merit Health Woman's Hospital in Flowood-Jackson, Mississippi, has demonstrated that procedures can be completed efficiently and safely. When compared to the same procedures performed via laparotomy, the time to complete surgical procedures robotically has not increased, but decreased. In addition, our already low surgical infection rate has dropped further, and patient satisfaction remains high. While this article does not specifically address potential healthcare cost differentials between open and robotic-assisted laparoscopic surgical cases, the shorter post-operative hospital stays and faster return-to-work times possible for patients benefitting from advanced minimally-invasive surgery must be factored into the debate.

\section{Acknowledgments}

None.

\section{Conflict of interest}

The authors declare that there is no conflict of interest.

\section{References}

1. Lenihan JP. Flight plan for robotic surgery credentialing: New AAGL guidelines. OBG Management 2014;26(11):44-48.

2. Payne J. Robotic hysterectomy. Kiawah Island, USA: The Callender Society; 2013.

3. Brooke Monroe RN, Director of Surgery, Jennifer Freeny RN. Infection Control Department, Merit Health; Woman's Hospital, December 2014.

4. Mary Chesnutt. RHIA, MHA, Chief Quality Officer, Merit Health Woman's Hospital, January 2015. 